\section{Comment on "Combining brentuximab vedotin with dexamethasone, high-dose cytarabine and cisplatin as salvage treatment in relapsed or refractory Hodgkin lymphoma: the phase II HOVON/LLPC Transplant BRaVE study."}

The high-dose cytarabine-containing regimen with dexamethasone, cytarabine and cisplatin (DHAP) had been predominantly used in Europe as standard salvage chemotherapy for relapsed or refractory (R/R) classical Hodgkin lymphoma (c-HL) until the recent introduction of brentuximab vedotin (Bv), an anti-CD30 antibody conjugated to the potent antimicrotubule agent monomethyl auristatin-E. ${ }^{1}$ Real-world experience shows a gradual transition from traditional cytotoxic agentbased treatment to selectively active agent-based treatment for R/R c-HL; ${ }^{2}$ in clinical practice, $\mathrm{Bv}$ was the first agent to be used for this purpose. However, the slight decline in the number of increased rates of metabolic complete response (mCR), as assessed by [18F] fluorodeoxyglucose (FDG)-positron emission tomography (PET)/computed tomography (CT) scan, after Bv exposure as single agent, has raised questions about its efficacy and widespread adoption in the R/R c-HL setting. ${ }^{2}$

We read with great interest the study by Kersten et al. published ahead of print in this journal. ${ }^{3}$ In their multicenter, international phase II study, between 2014 and 2017, 52 adult patients (median age: 29 years) with R/R $\mathrm{c}-\mathrm{HL}$ received $\mathrm{Bv}$ plus DHAP with the aim of testing the possibly enhanced cyto-destructive potency against Hodgkin and Reed-Sternberg (HRS) cells from this combination. Hospitalized patients received three 21-day cycles of Bv $1.8 \mathrm{mg} / \mathrm{kg}$ on day 1 and DHAP (dexamethasone $40 \mathrm{mg}$ on days $1-4$, cisplatin $100 \mathrm{mg} / \mathrm{m}^{2}$ in continuous intravenous infusion [i.v.] for 24 hours on day 1 and cytarabine $2 \times 2 \mathrm{~g} / \mathrm{m}^{2}$ ) on day 2 ; treatment-related adverse events were also evaluated. Despite several adverse prognostic factors (advanced-stage disease in $54 \%$ of patients, primary refractory disease in $43 \%$ of patients, and relapses within 1-year from first-line treatment in $29 \%$ of patients), Bv combined with DHAP resulted in $\mathrm{mCR}$ rates (according to the Lugano classification, 2014 criteria) of $81 \%$, and grade $\geq 3$ toxicity (prevalently consisting of febrile neutropenia according to the National Cancer Institute Common Terminology Criteria for Adverse Events [CTCAE], version 4.03) in only $22 \%$ of patients. Subsequently, 47 of 52 patients $(90 \%)$ received high-dose chemotherapy and hematopoietic stem cell transplantation (HSCT); after a median follow up of 27 months, the progression-free survival (PFS) was $74 \%$. The authors, supported by the evidence of a higher proportion of patients with $\mathrm{mCR}$ at 2 -years than traditional salvage regimens, concluded that, in R/R c-HL, three cycles of Bv-DHAP are impressively effective and greatly lower costs (using only 3 administrations of $\mathrm{Bv}$ ).

Several attempts have been made in the R/R c-HL setting to define the best partner(s) that could synergize with Bv. ${ }^{4-6}$ Clinical trials conducted in this setting present convincing evidence that increasing the dosage of bendamustine had good anti-cancer activity with no doselimiting toxicity. ${ }^{7}$ Improvements in anti-lymphomatous potency occurred especially when Bv infusion was followed by increasing doses of bendamustine, most likely due to an enhanced synergistic effect which was perceived as a great advantage in this subset. Emerging in vitro data suggest that high-dose bendamustine administered immediately after Bv facilitated intracellular traf- ficking, internalization, and metabolism of antiCD30auristatin conjugates, and thus targeted delivery of anticancer therapeutics. ${ }^{8}$ We have published ${ }^{9,10}$ encouraging preliminary single-center efficacy and safety results of a new salvage regimen named $\mathrm{Bv}+\mathrm{Bs}-21$ for $\mathrm{R} / \mathrm{R} \mathrm{c}-\mathrm{HL}$; this consists of 3-days outpatient i.v. infusions of $1.8 \mathrm{mg} / \mathrm{kg}$ of brentuximab vedotin on day 1 of each 3 -week cycle combined in sequence to bendamustine supercharge on days 2 and 3 of the treatment cycle at a fixed dose of 120 $\mathrm{mg} / \mathrm{m}^{2}$ per day, for a total of four courses. Mature results (in terms of larger patient numbers and longer follow-up than in the previously published study ${ }^{9,10}$ ) are now available. These are characterized by a prospective series of 30 patients (median age: 44 years; range: $23-59$ years) receiving $\mathrm{Bv}+\mathrm{Bs}-21$ for $\mathrm{R} / \mathrm{R}$ c-HL during the period from 2013 to 2020 at the Hematology Unit of the Federico II University of Naples, Italy, whose clinical presentations were more aggressive ( $>3$ lines of previous treatment in $75 \%$ of patients, primary refractory disease in $70 \%$ of patients, autologous HSCT failure in $35 \%$ of patients) compared to those in the Kersten et al. ${ }^{3}$ study. All patients underwent four courses of $\mathrm{Bv}+\mathrm{Bs}-21$; mediandose intensity during sequential therapy was $100 \%$ (range: 88.6-102.4\%) for $\mathrm{Bv}$ and $100 \%$ (range: 88.7 $102.4 \%$ ) for Bs. A vigorous support drug treatment (especially premedication prior to Bs administration, and antimicrobial drugs) and clinical and laboratory monitoring were systematically performed in all cases. Ten patients $(33 \%)$ experienced grade $\geq 3$ treatment-related adverse events (according to the CTCAE, version 4.03) consisting of cytomegalovirus reactivation (with viremia [median CMV-DNA: 1,810 IU/mL; range: 620-170,000 $\mathrm{IU} / \mathrm{mL}$ ] with fever, which was successfully treated with pre-emptive therapy with valganciclovir) in seven cases and neutropenia in three cases; all resolved without requiring hospitalization. At post-Bv+Bs-21 re-evaluation, $100 \%$ of patients had deep metabolic responses with Deauville 5 -point scale scores $\leq 3$ at FDG-PET/CT scans. Thereafter, four patients $(13 \%)$ received two additional courses of $\mathrm{Bv}+\mathrm{Bs}-21$, five patients (17\%) received allogeneic HSCT, and the remaining 21 patients $(70 \%)$ received autologous HSCT. In this last sub-group, for 12 patients, peripheral blood stem cells (PBSC) were previously harvested after two courses of ifosfamide, gemcitabine, vinorelbine and prednisolone; in the remaining nine cases, PBSC were successfully collected after $\mathrm{Bv}+\mathrm{Bs}-21$, with mobilization with G-CSF, vinorelbinecyclophosphamide and/or plerixafor regimen. The median peak value of $\mathrm{CD} 34^{+}$cells was reached on day 12 after mobilization (median number of harvested CD34 ${ }^{+}$cells: $3.1 \times 10^{6}$ per kilogram of body weight; range: 1.6$\left.4.2 \times 10^{6}\right)$. After HSCT, the median day of engraftment of neutrophils and platelets was recorded on day 11 (range: 9-21 days) and day 12 (range: 9-25 days), respectively. At a median follow up of 36 months (range: 1-83 months) from the end of the $\mathrm{Bv}+\mathrm{Bs}-21$ regimen, the estimated 3year PFS of the entire population was $94 \%$ (95\% confidence interval: $84.4-100 \%$ ).

In conclusion, our clinical data indicate that bendamustine (an old and low-cost cytotoxic agent) used in a new schedule modality (i.e., administered at increased dose and after the first-in-class antibody drug conjugate targeting CD30) has highly synergistic activity in outpatient salvage regimen against R/R HRS cells of patients aged $<60$ years.

\section{Marco Picardi and Claudia Giordano}

Department of Clinical Medicine and Surgery, Hematology Unit, Federico II University Medical School, Naples, Italy 
Correspondence: CLAUDIA GIORDANO -

claudiagiordano91@hotmail.com

doi:10.3324/haematol.2020.278203

Disclosures: no conflicts of interest to disclose.

Contributions: MP and CG performed research; MP wrote the manuscript; FP supervised the study.

\section{References}

1. Younes A, Bartlett NL, Leonard JP, et al. Brentuximab vedotin (SGN35) for relapsed CD30-positive lymphomas. N Engl J Med. 2010;363(19):1812-1821.

2. Connors JM. Hodgkin lymphoma: outsmarting HRS cells. Blood. 2020;136(21):2362-2364.

3. Kersten MJ, Driessen J, Zijlstra JM, et al. Combining brentuximab vedotin with dexamethasone, high-dose cytarabine and cisplatin as salvage treatment in relapsed or refractory Hodgkin lymphoma: the phase II HOVON/LLPC Transplant BRaVE study. Haematologica. 2020 Apr 9. [Epub ahead of print] doi:haematol.2019.243238.

4. Moskowitz AJ, Schröder H, Yahalom J, et al. PET-adapted sequential salvage therapy with brentuximab vedotin followed by augmented ifosfamide, carboplatin, and etoposide for patients with relapsed and refractory Hodgkin's lymphoma: a non-randomised, open-label, single-centre, phase 2 study. Lancet Oncol. 2015;16(3):284-292.

5. LaCasce AS, Bociek RG, Sawas A, et al. Brentuximab vedotin plus bendamustine: a highly active first salvage regimen for relapsed or refractory Hodgkin lymphoma. Blood. 2018;132(1):40-48.

6. Garcia-Sanz R, Sureda A, de la Cruz F, et al. Brentuximab vedotin and ESHAP is highly effective as second-line therapy for Hodgkin lymphoma patients (long-term results of a trial by the Spanish GELTAMO Group). Ann Oncol. 2019;30(4):612-620.

7. Cohen JB, Wei L, Maddocks KJ, et al. Gemcitabine and bendamustine is a safe and effective salvage regimen for patients with recurrent/refractory Hodgkin lymphoma: results of a phase 1/2 study. Cancer. 2020;126(6):1235-1242.

8. De Filippi R, Cillo M, Crisci S, et al. Continuous exposure to bendamustine (BDM) results in stable upregulation of CD30 and increased sensitivity to brentuximab vedotin (BV) in tumor cells of Hodgkin lymphoma (HL). Blood. 2015;126(23):2479.

9. Picardi M, Della Pepa R, Giordano C, et al. Brentuximab vedotin followed by bendamustine supercharge for refractory or relapsed Hodgkin lymphoma. Blood Adv. 2019:3(9):1546-1552.

10. Della Pepa R, Picardi M, Pugliese N, et al. Brentuximab vedotin followed by bendamustine supercharge for refractory or relapsed Hodgkin lymphoma: mature results of a monocentric prospective trial. Haematologica. 2020;105(s2):S101-102. 